PROCEEDINGS OF THE

AMERICAN MATHEMATICAL SOCIETY

Volume 134, Number 6 , Pages 1591-1598

S 0002-9939(05)08169-4

Article electronically published on December 2, 2005

\title{
THE DEPTH OF THE JACOBIAN RING OF A HOMOGENEOUS POLYNOMIAL IN THREE VARIABLES
}

\author{
ARON SIMIS \\ (Communicated by Bernd Ulrich)
}

\begin{abstract}
The question as to whether the Jacobian ideal of an irreducible projective plane curve always admits an irrelevant component has been going around for some years. One shows that a curve will satisfy this if it has only ordinary nodes or cusps, while an example is given of a family of sextic curves whose respective Jacobian ideals are saturated. The connection between this problem and the theory of homogeneous free divisors in three variables is also pointed out, so the example gives a family of Koszul-free divisors.
\end{abstract}

\section{Introduction: The PROBlem}

Let $k$ be an algebraically closed field of characteristic zero. Given a homogeneous polynomial $f \in R=k[x, y, z]$, let $I \subset R$ denote the ideal generated by the three partial derivatives of $f$ - called the gradient ideal of $f$.

It was 1976, I believe, when I first heard the following question from David Buchsbaum.

Question 1.1. Let $f$ be an irreducible polynomial of degree $\geq 3$. When is $\operatorname{depth}(R / I)=0$ ?

Since I was then looking at free resolutions and syzygetic ideals, it seemed like a natural problem to look at - which I did not. Sometime later, I discussed this question with Ernst Kunz. He suggested looking into the Hessian $H(f)$ of $f$ since $H(f) \in I:(x, y, z)$ from the Euler formula for $f$. Alas, very often $H(f) \in I$, thus yielding no information on the problem. It thus seemed that the problem deserved a harder look. Still later Karl-Otto Stöhr produced an argument which roughly showed that the problem ought to have an affirmative answer for very simple singularities. As far as I remember, that stayed at that. I believe none of us ever looked into it again. Unfortunately, I do not recall Stöhr's argument and I am afraid he may not recall it either after so many years.

As a way of paying tribute to all three fine mathematicians for their interest in the problem, though they may not have recollection of it after over 25 years, I will reprove the ordinary node case by a simple look at the Hilbert polynomial of $R / I$.

Received by the editors October 18, 2004 and, in revised form, January 6, 2005.

2000 Mathematics Subject Classification. Primary 13C14, 13C15, 13H10, 13D02, 13D40, 13H15; Secondary 12E05, 14B05, 14H50.

The author was partially supported by a CNPq grant and the Brazil-France Cooperation in Mathematics.

(C)2005 American Mathematical Society Reverts to public domain 28 years from publication 
The emphasis throughout this paper is on the irreducibility of $f$, since reduced non-irreducible forms $f$ such that $\operatorname{depth}(R / I)>0$ are easy to write down. Note that the question is easily answered if the corresponding projective plane curve has no singular points - in this case the gradient ideal is even $(x, y, z)$-primary. However, apart from this case there do not seem to be any totally obvious situations where the question has an immediate answer. One possibility is to compare the restriction, if any, imposed on the multiplicity of the Jacobian ring $R / I$ in order that its depth be zero, to the one imposed by the irreducibility of $f$.

Clearly, saying that $\operatorname{depth}(R / I)>0$ is tantamount in this simple setup to asking whether $I$ is a codimension two perfect ideal. Thus, in the present situation of a polynomial ring in three variables, the problem will tie up with the question of finding free homogeneous divisors in the sense of Saito (6]) (who called them logarithmic divisors in the complex analytic setup).

I am indebted to the referee for a fine work in pointing several imprecisions and also for correcting a wrong calculation in the first version, thus showing that the method employed in the proof of Proposition 2.1 is really tight.

For the sake of completeness and ready reference, I reprove the following wellknown formula by an elementary self-contained argument.

Lemma 1.2. Let $I \subset R$ be a perfect ideal (i.e., $R / I$ is Cohen-Macaulay) of codimension two, generated by three forms of equal degree $d \geq 2$. Then the Hilbert polynomial of $R / I$ is

$$
H(R / I)=\left(\begin{array}{c}
d+d_{0}-1 \\
2
\end{array}\right)+\left(\begin{array}{c}
2 d-d_{0}-1 \\
2
\end{array}\right)-3\left(\begin{array}{c}
d-1 \\
2
\end{array}\right)+1,
$$

for some $1 \leq d_{0} \leq\left\lfloor\frac{d}{2}\right\rfloor$.

Proof. The integer $d_{0}$ will be immediately clear from the argument. The minimal graded free resolution of $R / I$ as an $R$-module is of the form

$$
0 \longrightarrow R(-m) \oplus R(-n) \stackrel{\varphi}{\longrightarrow} R(-d)^{3} \longrightarrow R \longrightarrow R / I \longrightarrow 0,
$$

for suitable positive integers $m, n$. The Hilbert function of $R / I$ is then

$$
\begin{aligned}
H(R / I, t) & =H(R, t)-3 H(R, t-d)+H(R, t-m)+H(R, t-n) \\
& =\left(\begin{array}{c}
t+2 \\
2
\end{array}\right)-3\left(\begin{array}{c}
t-d+2 \\
2
\end{array}\right)+\left(\begin{array}{c}
t-m+2 \\
2
\end{array}\right)+\left(\begin{array}{c}
t-n+2 \\
2
\end{array}\right) .
\end{aligned}
$$

Since $\operatorname{dim} R / I=1$, the Hilbert polynomial is the constant integer $H(R / I)=$ $H(R / I, t)$ for $t>>0$. Therefore, one only has to compute the coefficient of the degree zero term in $t$. A computation then yields

$$
H(R / I)=\left(\begin{array}{c}
m-1 \\
2
\end{array}\right)+\left(\begin{array}{c}
n-1 \\
2
\end{array}\right)-3\left(\begin{array}{c}
d-1 \\
2
\end{array}\right)+1 .
$$

Finally, by the Hilbert-Burch result, $I$ is generated by the maximal (i.e., $2 \times 2$ ) minors of a homogeneous $2 \times 3$ matrix representing $\varphi$. Therefore, if one sets $m=$ $d+d_{0}, n=d+d_{1}$, then it must be the case that $d=d_{0}+d_{1}$. Substituting for these values in (3), one gets the desired expression. 
Corollary 1.3. Let $f \in R$ be a squarefree homogeneous polynomial of degree $d+1 \geq$ 3 and let $I \subset R$ denote its gradient ideal. If $\operatorname{depth}(R / I)>0$, then

$$
\operatorname{deg}(R / I)=\left(\begin{array}{c}
d+d_{0}-1 \\
2
\end{array}\right)+\left(\begin{array}{c}
2 d-d_{0}-1 \\
2
\end{array}\right)-3\left(\begin{array}{c}
d-1 \\
2
\end{array}\right)+1,
$$

for some $1 \leq d_{0} \leq\left\lfloor\frac{d}{2}\right\rfloor$.

Remark 1.4. The above degree is what is called the (algebraic) multiplicity of the graded ring $R / I$ or, what is the same, the Hilbert-Samuel multiplicity of the localization of $R / I$ at the maximal ideal $(x, y, z)$. It is also the degree of the projective scheme associated to the ideal $I$ - to be distinguished from the degree of the zero-dimensional algebraic set $V(I) \subset \mathbb{P}^{2}$ which is the algebraic multiplicity of the radical of $I$.

\section{Affirmative ANSWER FOR ORDinARY NODES}

Here is a fair positive case of the question above.

Proposition 2.1. Let $f$ be irreducible of degree $d+1 \geq 3$ admitting only ordinary double points. Then $\operatorname{depth}(R / I)=0$, where $I$ is the gradient ideal of $f$.

Proof. Since $f$ is irreducible, one has the known inequality (see, e.g., 4, Lemma 18.4])

$$
\left(\begin{array}{l}
d \\
2
\end{array}\right) \geq \sum_{P}\left(\begin{array}{c}
m_{P} \\
2
\end{array}\right)
$$

where $m_{P}$ denotes the multiplicity of the curve defined by $f$ at the point $P \in \mathbb{P}^{2}$. Now, the right-hand side of (5) is really the number of singular points of the curve, since they are all ordinary double points.

Let $P \in \mathbb{P}^{2}$ be a singular point of the curve. Denote by $\wp$ the prime ideal of $P$ in $R$ and by $\mathcal{P}$ the $\wp$-primary component of $I$.

I claim that the $R_{\wp}$-module $R_{\wp} / I_{\wp}=R_{\wp} / \mathcal{P}_{\wp}$ has length one. For this, one can pass to an affine piece while keeping the same notation. Thus, up to a projective coordinate change, now $R=k[X, Y](X=x / z, Y=y / z), \mathcal{P}$ is primary to the maximal ideal $(X, Y)$, and the latter is the ideal of an ordinary double point of the corresponding affine piece of the curve. The affine equation of the curve can be taken to be of the form $f=X^{2}-Y^{2}+g(X, Y)$, where $g$ has initial degree at least 3 . One has to show that $\ell\left(k[X, Y]_{(X, Y)} / I_{(X, Y)}\right)=1$, where $\ell(-)$ denotes length.

This is a standard calculation in plane curve theory. For the sake of completeness, I give the following elementary argument. By Euler's formula, $I$ is generated in this affine piece by the polynomials $f, \partial f / \partial x, \partial f / \partial y$. Write $\partial g / \partial X=X h_{1}(X, Y)+$ $h_{2}(Y)$, where $h_{1}(0,0)=0$ and $h_{2} \in k[Y]$ has initial degree at least 2. Then

$$
\partial f / \partial X=X\left(2+h_{1}(X, Y)\right)+h_{2}(Y),
$$

with $2+h_{1}$ a unit locally at $(X, Y)$. Therefore, for the sake of generating the ideal locally at $(X, Y)$, the partial derivative looks like $X-u F(Y)$, with $u$ a unit and $F$ of initial degree at least 2. By the same token, the second partial derivative looks like $Y-v G(X)$ in the localization, with $G$ of initial degree at least 2. This shows 
that, locally at $(X, Y)$,

$$
k[X, Y] / I \simeq k[Y] /(Y-v G(u F(Y)), f(u F(Y), Y)) \simeq k[Y] /(Y, f(u F(Y)),
$$

where $f(u F(Y), Y) \in(Y)$. Therefore, $k[X, Y]_{(X, Y)} / I_{(X, Y)}=k$, thus showing that the length is indeed one.

Next, up to a projective coordinate change, one may assume that the singular points of the curve all lie in one and the same affine piece. With this proviso, the original degree in the graded setup can be computed as the present length $\ell(R / I)$. By well-known properties of Artinian rings (see, e.g., 2, Corollary 2.16]), one has $\ell(R / I)=\sum_{i} \ell\left(R_{\wp_{i}} / I_{\wp_{i}}\right)$, where $i$ runs through the set indexing the minimal primes of $R / I$ (i.e., the finitely many singular points of the curve). Consequently, $\ell(R / I)$ is also the number of singular points of the curve and so is the original graded degree.

Going back to the original notation in the graded setup, if one assumes that the depth of $R / I$ is positive, then, by Corollary 1.3 and (5), the following inequality holds:

$$
\left(\begin{array}{l}
d \\
2
\end{array}\right) \geq\left(\begin{array}{c}
d+d_{0}-1 \\
2
\end{array}\right)+\left(\begin{array}{c}
2 d-d_{0}-1 \\
2
\end{array}\right)-3\left(\begin{array}{c}
d-1 \\
2
\end{array}\right)+1
$$

Arguing that this is impossible will lead to the required contradiction. Expanding all binomial terms in the inequality yields

$d(d-1) \geq\left(d+d_{0}-1\right)\left(d+d_{0}-2\right)+\left(2 d-\left(d_{0}+1\right)\right)\left(2 d-\left(d_{0}+2\right)\right)-3(d-1)(d-2)+2$,

which, after collecting terms and simplifying, is equivalent to $\left(d-d_{0}\right)^{2}+d+d_{0}^{2} \leq 0$ - clearly, absurd for positive values of $d$.

Remark 2.2. Though the inequality $\left(d-d_{0}\right)^{2}+d+d_{0}^{2} \leq 0$ fails in quite a slack way, the method of proof does not leave much room for other situations such as, e.g., when the curve has only ordinary nodes and ordinary cusps. Indeed, in this case a similar local analysis of the singularities yields that $\operatorname{deg}(R / I)$ is at most twice the number of singular points. In the worst case, this would lead one to multiply the left-hand side of (6) by 2 and still try to show it fails. However, the value $d_{0}=2$ already makes the inequality kosher for $d \geq 4$. On the bright side, however, this is in general not the inequality to be contradicted when $R / I$ fails to be Cohen-Macaulay. In such cases, one would have to deal directly with $\operatorname{deg}(R / I)$ in order to derive the impossibility of equality (4). The problem is roughly how $\operatorname{deg}(R / I)$ grows in the presence of singularities so one can compare it directly to the right-hand side of (6). Thus, the sole hypothesis of irreducibility may in general fall short of implying that $\operatorname{depth}(R / I)=0$. Indeed, an example of such a phenomenon will be given in the next section.

\section{Counterexample: The family of Cayley sextics}

Consider a cubic equation with parametric coefficients $T^{3}-p T+q$, where $p, q$ are fixed polynomials in $B=k[u, v, x, y, z]$. Here one takes very special $p, q$, namely, $p=x^{2}+v y^{2}+u x z$ and $q=u\left(x^{2}+v y^{2}\right) z$. Then $T^{3}-p T+q$ is a quasi-homogenous polynomial of degree 6 with respect to the following weights:

$$
\begin{array}{ll}
\operatorname{deg} u=1, & \operatorname{deg} x=2, \\
\operatorname{deg} v=2, & \operatorname{deg} y=1, \\
\operatorname{deg} T=2, & \operatorname{deg} z=1 .
\end{array}
$$


Thus, the discriminant of $T^{3}-p T+q$ is a quasi-homogeneous polynomial of degree 12

$$
F=F(u, v, x, y, z)=4\left(x^{2}+v y^{2}+u x z\right)^{3}-27 u^{2}\left(x^{2}+v y^{2}\right)^{2} z^{2},
$$

which gives a family of quasi-homogeneous surfaces in the affine space $\mathbb{A}^{3}$ with weights $2,1,1$ over the affine plane $\mathbb{A}^{2}$ with weights 1,2 — called the Cayley family.

Now, for every $(a, b) \in \mathbb{A}^{2}$ such that $a b \neq 0$, the corresponding fiber gives a form $f=f_{a, b} \in k[x, y, z]$ of (standard) degree 6 . Such an individualized form is called a Cayley sextic.

I will use the following elementary result, a possibly well-known variation of irreducibiliy criteria. A more general statement is certainly available, but there is no need for it in the present situation.

Lemma 3.1 ( $k$ algebraically closed). Let $P(y, z)=z^{3}+f_{1}(y) f_{2}(y) z^{2}+c f_{1}(y)^{2} z+$ $d f_{1}(y)^{3} \in k[y, z]$, where $f_{1}(y), f_{2}(y)$ are non-zero polynomials of degree 2 and $c, d$ are non-zero elements of $k$. Then $P(y, z)$ is irreducible in $k[y, z]$.

Proof. If it factors one must have $P(y, z)=(z+g(y))\left(z^{2}+h_{1}(y) z+h_{2}(y)\right)$, where $g(y), h_{1}(y), h_{2}(y) \in k[y]$. Expanding this product and observing that $f_{1}(y)$ is a common factor of all $y$-coefficients of $P(y, z)$ except the first, it follows that a prime factor of $f_{1}(y)$ divides all three $g(y), h_{1}(y), h_{2}(y)$. Now, $h_{1}(y) \neq 0$, as otherwise $g(y)$ and $h_{2}(y)$ would both have to be of degree 4 to comply with $y$-degrees of $P(y, z)$, which is impossible since their product has degree 6 . Therefore, $\operatorname{deg} h_{1}(y) \geq 1$.

This then rules out the possibility that $\operatorname{deg} g(y) \geq 4$, as otherwise $\operatorname{deg} h_{2}(y) \leq 2$, which would entice that the $y$-coefficient of $z$ in $P(y, z)$ have the wrong degree. Thus, $1 \leq \operatorname{deg} g(y) \leq 3$, since $g(y)$ has a prime factor. In this range, necessarily $\operatorname{deg} h_{1}(y)=4$ in order to comply with the degree of the $y$-coefficient of $z^{2}$ in $P(y, z)$; therefore, $5 \leq \operatorname{deg} g(y) h_{1}(y) \leq 7$. To have cancelation in the $y$-coefficient of $z$ in $P(y, z)$, one would need $\operatorname{deg} h_{2}(y) \geq 5$. The only way would be to have $\operatorname{deg} g(y)=1, \operatorname{deg} h_{2}(y)=5$.

In this case, there is no apparent degree contradiction, so one resorts to looking closely at the coefficients of the resulting expansion of the product of the two factors. Note that, necessarily, $g(y)$ is a factor of both $h_{1}(y)$ and $h_{2}(y)$, so write $h_{1}(y)=g(y) h_{1}^{\prime}(y)$ and $h_{2}(y)=g(y) h_{2}^{\prime}(y)$. The following polynomial emerges:

$$
z^{3}+g(y)\left(1+h_{1}^{\prime}(y)\right) z^{2}+g(y)\left(g(y) h_{1}^{\prime}(y)+h_{2}^{\prime}(y)\right) z+g(y)^{2} h_{2}^{\prime}(y) .
$$

Also recall that $g(y)$ is now a prime factor of $f_{1}(y)$, so $f_{1}(y)=g(y) g^{\prime}(y)$, where $g^{\prime}(y)$ is the other factor. Substituting and comparing with the given $y$-coefficients of 1 and of $z$ in $P(y, z)$, one finds $h_{2}^{\prime}(y)=d g(y) g^{\prime}(y)^{3}$ and

$$
\begin{aligned}
c g(y)^{2} g^{\prime}(y)^{2} & =g(y)\left(g(y) h_{1}^{\prime}(y)+h_{2}^{\prime}(y)\right) \\
& =g(y)\left(g(y) h_{1}^{\prime}(y)+d g(y) g^{\prime}(y)^{3}\right) \\
& =g(y)^{2}\left(h_{1}^{\prime}(y)+d g^{\prime}(y)^{3}\right),
\end{aligned}
$$

hence $g^{\prime}(y)$ divides $h_{1}^{\prime}(y)$. Comparing with the $y$-coefficient of $z^{2}$, one gets

$$
f_{2}(y) g(y) g^{\prime}(y)=g(y)\left(1+h_{1}^{\prime}(y)\right),
$$

from which it is seen that $g^{\prime}(y)$ divides $1+h_{1}^{\prime}(y)$ - absurd.

This concludes the proof. 
Corollary 3.2. A Cayley sextic is irreducible.

Proof. It suffices to show the irreducibility of the affine piece at $x=1$. The latter can be seen to be

(7) $f_{a, b}(1, y, z)=z^{3}-\frac{3}{4}\left(9 b y^{2}+5\right)\left(\frac{b}{a} y^{2}+\frac{1}{a}\right) z^{2}+3\left(\frac{b}{a} y^{2}+\frac{1}{a}\right)^{2} z+\left(\frac{b}{a} y^{2}+\frac{1}{a}\right)^{3}$.

Apply the previous lemma with $f_{1}(y)=\frac{b}{a} y^{2}+\frac{1}{a}$ and $f_{2}(y)=-\frac{3}{4}\left(9 b y^{2}+5\right)$.

Remark 3.3. It is also possible to find the singular points of a Cayley sextic and their respective multiplicities and then proceed to show that it is rational, hence irreducible. However, I find the previous approach more elementary.

Now it is time to prove that the Jacobian ring $R / I$ of the Cayley sextic has positive depth, in other words, that $I$ is a (codimension two) perfect ideal. Since the intention is to do slightly more, I will start a new section. The required result will be part of Proposition 4.4. where it will also be expressed in the language of free divisors in order to point a different facet of the problem.

\section{Relation to THE THEORY OF FREE Divisors}

Keep the previous notation, except that $J_{f}$ will now denote the gradient ideal of $f$. The purpose of this section is to prove that the Jacobian ideal of any Cayley sextic is a codimension two perfect ideal, while relating the main problem to the theory of free divisors. Recall some of the features of this notion (see 6, also 7]).

Let $f \in R$ be a squarefree polynomial. $f$ will be said to be a divisor of linear type if $J_{f}$ is an ideal of linear type. Following the idea of Saito $([\underline{6})$, one says that $f$ is a free divisor if $\operatorname{Der}_{f}(R):=\left\{\delta \in \operatorname{Der}_{k}(R) \mid \delta(f) \in(f)\right\}$ - the $(f)$-idealizer of $\operatorname{Der}_{k}(R)$-is a free $R$-module.

In dimension three, there is the following special result.

Proposition 4.1. Let $f \in R=k[x, y, z]$ be a squarefree homogeneous polynomial of degree $\geq 3$. The following are equivalent:

(i) $J_{f}$ is a codimension two perfect ideal,

(ii) $f$ is a free divisor.

This is a special case of a general structure result of free divisors which are not smooth everywhere. For a simple proof see [7, Proposition 3.7], but the priority of this result is Terao's.

The question in the first section can be rephrased in the present terminology.

Question 4.2. What are the irreducible free homogeneous divisors $f \in k[x, y, z]$ of degree $\geq 3$ ?

Remark 4.3. There are many irreducible homogeneous $3 \times 3$ determinantal polynomials $f$-any randomly chosen $3 \times 3$ graded matrix over the standard graded polynomial ring $k[x, y, z]$ will yield such a polynomial. However, in order for $f$ to be moreover a free divisor, it is required that such a $3 \times 3$ graded matrix have the following form up to $k$-linear elementary transformations:

$$
\frac{1}{\operatorname{deg}(f)}\left(\begin{array}{ccc}
x & p_{1}(x, y, z) & q_{1}(x, y, z) \\
y & p_{2}(x, y, z) & q_{2}(x, y, z) \\
z & p_{3}(x, y, z) & q_{3}(x, y, z)
\end{array}\right),
$$


where the partial derivatives of $f$ are the signed cofactors of $x, y, z$, respectively. For further assistance on these matters, see [7, Supplement to Proposition 3.7].

It is convenient to extend the previous notions to families of divisors. If $S=k[\mathbf{u}]$ is a second polynomial ring over $k$ and $F \in S \otimes_{k} R \simeq B=k[\mathbf{u}, \mathbf{x}]$ has unit $\mathbf{u}$-content, then one speaks of $F$ as a family of divisors of linear type if the relative gradient ideal $J_{F, \mathbf{x}} \subset B$ ( $\mathbf{x}$-derivatives only) is an ideal of linear type.

One can also speak of families of free divisors. For this, let $\operatorname{Der}_{F, \mathbf{x}}(B) \subset \operatorname{Der}_{\mathbf{x}}(B)$ stand for the $(F)$-idealizer of $\operatorname{Der}_{\mathbf{x}}(B)$ (i.e., the analogue of the modules $\operatorname{Der}_{f}(R) \subset$ $\operatorname{Der}(R)$ with respect to the $\mathbf{x}$-derivatives only). Then, by a similar token, $F$ will be said to be a family of free divisors, provided $\operatorname{Der}_{F, \mathbf{x}}(B)$ is a free $B$-module of rank $n$.

Now let $F$ and $f$ denote, respectively, the Cayley family and any Cayley sextic. Since $F$ has unit $u, v$-content and $f$ is irreducible over $k[x, y, z], F$ is irreducible over $B=k[u, v, x, y, z]$. Furthermore:

Proposition 4.4. Let $F$ denote the Cayley family and let $f$ stand for any of its Cayley sextics. Then:

(i) $J_{F, \mathbf{x}}$ and $J_{f}$ are both codimension two perfect ideals,

(ii) $F$ is a (quasi-homogeneous) family of free divisors,

(iii) $f$ is a (homogeneous) free divisor of linear type.

Proof. (i) A calculation with Macaulay ([1]) yields that the relative gradient ideal $J_{F, \mathbf{x}}$ is a codimension two perfect ideal with Hilbert-Burch matrix

$$
\mathcal{M}=\left(\begin{array}{lr}
u x^{2}-7 u v y^{2}-2 u^{2} x z & v x^{2} y+v^{2} y^{3}-u v x y z \\
8 u x y-u^{2} y z & -x^{3}-v x y^{2}+1 / 2 u x^{2} z-1 / 2 u v y^{2} z \\
-2 x^{2}-2 v y^{2}-6 u x z+2 u^{2} z^{2} & u v y z^{2}
\end{array}\right) .
$$

(If a computational argument is hard to swallow, one may laboriously check by hand that the three $2 \times 2$-minors of $\mathcal{M}$ coincide with the partial derivatives up to non-zero factors from $k$.)

As for $f$, since it is the image of $F$ by a ring homomorphism $k[u, v, x, y, z] \rightarrow$ $k[x, y, z]$, its partial derivatives are specialized from the $x, y, z$ partial derivatives of $F$. Further, since the latter are the (maximal) minors of a matrix, then the partial derivatives of $f$ are the (maximal) minors of the specialized matrix. Since $f$ is irreducible, the gradient ideal $J_{f}$ has codimension two, hence it is a perfect ideal.

(ii) The analogue of the exact sequence (7) in [7, Proposition 3.2] holds in the present relative context, as one readily sees. Since $F$ is quasi-homogeneous, it follows that $\operatorname{Der}_{F, \mathbf{x}}(B)$ is the free $B$-module generated by the columns of this matrix and by the Euler vector $(x, y, z)^{t}$. This shows that $F$ is a family of free divisors by the above definition.

(iii) The argument to show that $f$ is a free divisor is similar (more directly, use Proposition 4.1).

To see that $f$ is a divisor of linear type, it suffices by [5, Lemma 8.2, Theorem 9.2 and Corollary 10.4] to verify that, after specializing, the entries of $\mathcal{M}$ generate an $(x, y, z)$-primary ideal. For that, it suffices to check that $I_{1}(\mathcal{M}):(u v)^{\infty}$ has codimension three. Again a computation with Macaulay will readily fill this intent, or else, by direct inspection of the entries of $\mathcal{M}$ while pretending that $u, v$ are units. 
Remark 4.5. $F$ is actually a family of Koszul-free divisors. The definition of a Koszul-free divisor in the complex analytic setup was originally given in [3]. For a slightly different treatment in the polynomial setup see [7. According to the latter, any free quasi-homogeneous divisor in three variables is Koszul free.

Let me note that $F$ is not of linear type (as a divisor in 5 -affine space). However, $F R_{u}$ is of linear type as a divisor in the ring of fractions $R_{u}$ (i.e., the extended Jacobian ideal $I_{F} R_{u}$ is of linear type).

\section{REFERENCES}

[1] D. Bayer and M. Stillman, Macaulay: a computer algebra system for algebraic geometry, Macaulay version 3.01994 (Macaulay for Windows by Bernd Johannes Wuebben, 1996).

[2] D. Eisenbud, Commutative Algebra with a view toward Algebraic Geometry, Springer-Verlag, Berlin, Heidelberg, New York, 1995. MR.1322960 (97a:13001)

[3] F. J. Calderón-Moreno and L. Narváez-Macarro, The module $\mathcal{D} f^{s}$ for locally quasihomogeneous free divisors, Compos. Math. 134, No.1, (2002), 59-74. MR1931962 (2003i:14016)

[4] G. G. Gibson, Elementary Geometry of Algebraic Curves: an Undergraduate Introduction, Cambridge University Press, Cambridge, 1998. MR1663524 (2000a:14002)

[5] J. Herzog, A. Simis, and W. Vasconcelos, Koszul homology and blowing-up rings, in Commutative Algebra, Proceedings: Trento 1981 (S. Greco and G. valla, Eds.), Lecture Notes in Pure and Applied Mathematics 84, Marcel-Dekker, New York, 1983, pp. 79-169. MR0686942 (84k:13015)

[6] K. Saito, Theory of logarithmic differential forms and logarithmic vector fields, J. Fac. Sci. Univ. Tokyo Sect. 1A Math. 27 (1980), 265-291. MR0586450 (83h:32023)

[7] A. Simis, Differential idealizers and algebraic free divisors, in Commutative Algebra with A focus on GeOmetric And homological aspects, Proceedings of Sevilla, June 18-21, 2003 and Lisbon, June 23-27, 2003, Lecture Notes in Pure and Applied Mathematics, Marcel Dekker, vol. 244, 2005, pp. 211-226.

Departamento de Matemática, CCen, Universidade Federal de Pernambuco, Cidade UNIVERsitária, 50740-540 RECIFE, PE, BrazIL

E-mail address: aron@dmat.ufpe.br 